\section{Disturbance Analysis of Nonlinear Differential Equation Models of Genetic SUM Regulatory Networks}

\author{
Ping Li and James Lam
}

\begin{abstract}
Noise disturbances and time delays are frequently met in cellular genetic regulatory systems. This paper is concerned with the disturbance analysis of a class of genetic regulatory networks described by nonlinear differential equation models. The mechanisms of genetic regulatory networks to amplify (attenuate) external disturbance are explored, and a simple measure of the amplification (attenuation) level is developed from a nonlinear robust control point of view. It should be noted that the conditions used to measure the disturbance level are delay-independent or delay-dependent, and are expressed within the framework of linear matrix inequalities, which can be characterized as convex optimization, and computed by the interior-point algorithm easily. Finally, by the proposed method, a numerical example is provided to illustrate how to measure the attenuation of proteins in the presence of external disturbances.
\end{abstract}

Index Terms-Disturbance attenuation, asymptotic stability, genetic regulatory network, systems biology, time delay.

\section{INTRODUCTION}

SINCE the days of Nobert Wiener, system-level understanding has been a recurrent theme in biological sciences [1]. Traditionally, the different phases involved in the cellular processes were analyzed and characterized in isolation by using biochemical techniques, leading to the view that they operate independently. As systems biology emerges in the postgenomic era, one of the major challenges in contemporary systems biology has been to understand the gene regulation and function at the system level, for instance, how proteins are synthesized from genes as transcription factors binding to other genes, how DNA, RNA, and proteins interact with each other and other small molecules to coordinate multiple biological functions. These molecules and their interactions compose a complex network, known as genetic regulatory network (or simply, gene network).

Meanwhile, the development of modeling techniques has made it possible to apply mathematical methods to describe the network structure and predict the dynamic behavior of the genetic regulatory networks [2], [3], [4], and a large variety of formalisms have been proposed to model, analyze, and simulate genetic regulatory networks, such as directed graphs, Bayesian networks, Boolean networks, differential equations, and Petri nets (see also [5] and references therein for a wider categorization of gene network models). Although many approaches have been proposed to specify the gene network structure, it is generally accepted that finding the network topology is not sufficient to understand the network dynamics. In the case of differential equation models, the variables represent the concentrations of gene products, such as mRNAs, proteins, and other small molecules, as time-dependent values of the gene networks. Moreover, it is observed that genes spend a lot of time at intermediate values, which implies that gene expression levels tend to be continuous rather than binary [6]. The advantage of the description with differential equation networks is

- The authors are with the Department of Mechanical Engineering, The University of Hong Kong, Pokfulam Road, Hong Kong.

E-mail: \{pingli, james.lam\}@hku.hk.

Manuscript received 21 May 2009; revised 8 Sept. 2009; accepted 11 Sept. 2009; published online 10 May 2010.

For information on obtaining reprints of this article, please send e-mail to: tcbb@computer.org, and reference IEEECS Log Number TCBB-2009-05-0080. Digital Object Identifier no. 10.1109/TCBB.2010.19. that one can take into account detailed information about genetic regulatory mechanisms such as individual kinetics and interactions among mRNAs and proteins. In addition, it is also indispensable to study the genetic regulatory systems from the viewpoint of nonlinear system theory due to the fact that GRNs are strongly nonlinear and high dimensional.

In addition, it is recognized that it is system dynamics and internal structures of the biological phenomena that give rise to the functioning and function of cells [7]. In fact, stability is a fundamental requirement of biological systems, with obvious biological significance, see [8], [9] and references therein. In [10], the authors investigated a simple genetic circuit model in Escherichia coli to test the role of negative feedback in the stability analysis of gene networks. Also, an important issue in modeling gene expression is the fact that individual processes need a certain amount of time to be finished [11], [12]. This motivates to consider the effect of delay on dynamic behaviors of gene network, it has been well known that delay is often the key factor to instability of a given system, and thus, plays an important role in the dynamic analysis of gene regulation [13]. A nonlinear model of genetic regulatory networks with time delays was proposed, and sufficient conditions were also obtained in the form of linear matrix inequality in [14], where the transcription functions are assumed to act additively to regulate a gene. Such a regulatory function is known to be SUM logic [15], [16], which was first proposed in [17]. Sufficient conditions for the stochastic stability of the genetic networks with disturbance attenuation were also derived in [18], [19].

On the other hand, due to the fact that a realistic gene network model should be identified from real-world gene expression timeseries data, it is well known that the modeling error is unavoidable in practice, which makes the mathematical model uncertain. Moreover, one of the objectives of modeling is to obtain information about the input of the genetic regulatory networks, such as physical and chemical stimuli, and environmental changes [20]. It should be noted that the nature of these inputs and their corresponding values may not be fully known or measurable due to the complexity of biological processes [21]. When referring to modeling genetic regulatory networks, it is very difficult to fully detect the extrinsic signals, not to mention the fact that it is impossible to completely know the intrinsic inputs affecting each components of the gene network.

Motivated by the aforementioned reasons, in this paper, we propose a genetic regulatory network model with time delays and external disturbances, and investigate the dynamic performances of genetic regulatory networks. To be specific, the effects due to delays and external disturbances on the stability of gene networks are studied, sufficient conditions, which are delay-dependent or delay-independent, are established to measure the external disturbance level by means of nonlinear control theory. Moreover, an LMI optimization problem is further established to minimize the attenuation level of disturbances. It should be noted that the conditions obtained in this paper are expressed in a unified linear matrix inequality framework, and can be verified easily by existing standard software.

The remaining portion of this paper is organized as follows: Section 2 gives a system-level description on GRNs and presents the problem formulation. Section 3 is devoted to the stability analysis of the gene network in the presence of both time delays and external disturbances. A three-gene network is provided to show the effectiveness and applicability of the theoretical results in Section 4. In Section 5, we summarize our results.

Notations. Denote $\mathbb{Z}_{n} \triangleq\{1,2, \ldots, n\}$; let $\mathbb{R}$ be the set of real numbers; $\mathbb{R}^{n}$ denotes the $n$-column vectors; $\mathbb{R}^{m \times n}$ is the set of $m \times n$ matrices for which all components belong to $\mathbb{R}$. For any real symmetric matrices $P$ and $Q$, the notation $P \geq Q$ (respectively, $P>Q$ ) means that the matrix $P-Q$ is positive 


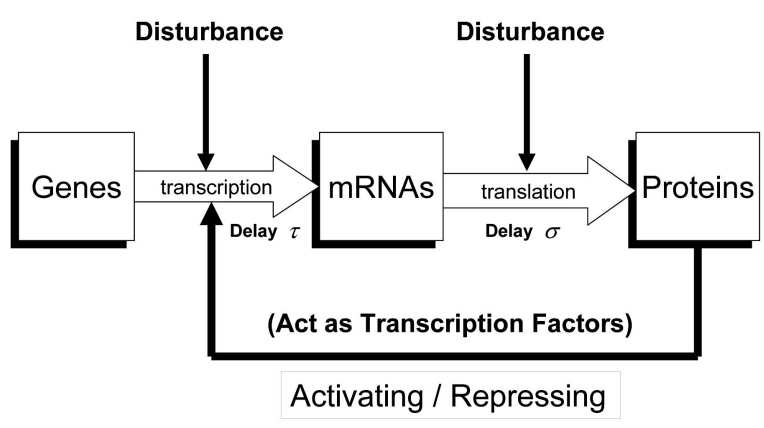

Fig. 1. System-level approach to GRNs.

semidefinite (respectively, positive definite). $|\cdot|$ denotes the euclidean norm for vectors and $\|\cdot\|$ denotes the spectral norm for matrices. $\mathcal{C}\left([-\tau, 0], \mathbb{R}^{n}\right)$ and $\mathcal{C}\left([-\tau, 0], \mathbb{R}_{+}^{n}\right)$ denote the family of continuous functions $\phi$ from $[-\tau, 0]$ to $\mathbb{R}^{n}$ and $\mathbb{R}_{+}^{n}$, with norm $|\phi|_{\tau}=\sup _{-\tau<s<0}|\phi(s)| . \operatorname{diag}(\ldots)$ stands for a block-diagonal matrix. The superscript " $T$ " denotes matrix transpose and the symbol \# is used to represent a matrix which can be inferred by symmetry. Matrices, if their dimensions are not explicitly stated, are assumed to have compatible dimensions for algebraic operations.

\section{Genetic Regulatory Network and Model DESCRIPTION}

\subsection{Genetic Regulatory network}

In living organisms, gene expression is regulated by genetic regulatory networks with interactions among DNA, RNA, proteins, and small molecules. In general, the term gene refers to those segments of one strand of DNA where genetic information can be transferred to mRNAs in a process called transcription. mRNA is then used as a template to synthesize proteins in a process called translation. In turn, some of proteins, acting as transcription factors, regulate the transcription process.

In addition, time delays and noise disturbances are common and substantial in the signal transmission along the pathway of gene networks. It is well known that gene transcription and mRNA translation must take some time to generate the corresponding product. Also, noise disturbances are always present during the process of gene expression, which are usually generated by its external environment. The whole scenario is shown from a system level point of view in Fig. 1.

\subsection{Problem Formulation}

The following differential equations have been used recently to describe the genetic regulatory networks [8], [22]:

$$
\left\{\begin{aligned}
\frac{\mathrm{d} m_{i}(t)}{d t}= & -a_{i} m_{i}(t)+f_{i}\left(p_{1}(t-\tau), p_{2}(t-\tau),\right. \\
& \left.\ldots, p_{n}(t-\tau)\right)+u_{i}, \\
\frac{\mathrm{d} p_{i}(t)}{d t}= & -b_{i} p_{i}(t)+d_{i} m_{i}(t-\sigma), i \in \mathbb{Z}_{n},
\end{aligned}\right.
$$

where $m_{i}(t)$ and $p_{i}(t)$ are the concentrations of the $i$ th mRNA and protein, and $a_{i}>0$ and $b_{i}>0$ are constant numbers, representing the degradation rate of the $i$ th mRNA and protein, respectively. $d_{i}>0$ is the production constant, and $u_{i}$ is defined as a basal rate, which may be considered as the "leakiness" of the promoter. In this paper, the function $f_{i}$ is taken as $f_{i}\left(p_{1}(t), p_{2}(t), \ldots, p_{n}(t)\right)=\sum_{j=1}^{n} f_{i j}\left(p_{j}(t)\right)$, which is called SUM logic, since each transcription factor acts additively to regulate the gene $i$. Here, $f_{i j}(\cdot)$ is a monotonic function of the Hill form, that is,

$$
f_{i j}=\left\{\begin{array}{cl}
\alpha_{i j} \frac{\left(x / \beta_{j}\right)^{H_{j}}}{1+\left(x / \beta_{j}\right)^{H_{j}}}, & \text { if transcription factor } j \\
\alpha_{i j} \frac{1}{1+\left(x / \beta_{j}\right)}, & \text { is an activator of gene } i, \\
\text { is anscription factor } j
\end{array}\right.
$$

where $H_{j}$ is the Hill coefficient, $\beta_{j}>0$ is a scalar, and $\alpha_{i j}$ is a bounded constant, which denotes the dimensionless transcriptional rate of transcription factor $j$ to gene $i$. Such a SUM logic is indeed exhibited in many natural gene networks [15], [16], [23], [24]. Note that when $n=1$, (1) degenerates into a single-gene network model, which has been proposed and investigated in [13]. Note

$$
\alpha_{i j} \frac{\left(x / \beta_{j}\right)^{H_{j}}}{1+\left(x / \beta_{j}\right)^{H_{j}}}=\alpha_{i j}\left(1-\frac{1}{1+\left(x / \beta_{j}\right)^{H_{j}}}\right),
$$

then, based on (2) and (3), the gene network in (1) can be rewritten as

$$
\left\{\begin{array}{l}
\frac{\mathrm{d} m_{i}(t)}{d t}=-a_{i} m_{i}(t)+\sum_{j=1}^{n} w_{i j} g_{j}\left(p_{j}(t-\tau)\right)+l_{i}, \\
\frac{\mathrm{d} p_{i}(t)}{d t}=-b_{i} p_{i}(t)+d_{i} m_{i}(t-\sigma), i \in \mathbb{Z}_{n},
\end{array}\right.
$$

where

$$
g_{j}(x)=\frac{\left(x / \beta_{j}\right)^{H_{j}}}{1+\left(x / \beta_{j}\right)^{H_{j}}}\left(j \in \mathbb{Z}_{n}\right), l_{i}=u_{i}+\sum_{j \in \mathcal{V}_{i}} \alpha_{i j}
$$

with $\mathcal{V}_{i}$ being the set of all the transcription factor $j$ which is a repressor of gene $i ; W=\left(w_{i j}\right)_{n \times n}$ is defined as follows: if transcription factor $j$ is an activator of gene $i, w_{i j}=\alpha_{i j}$; if there is no connection between $j$ and $i, w_{i j}=0$; if transcription factor $j$ is a repressor of gene $i, w_{i j}=-\alpha_{i j}$.

In this paper, we introduce external disturbances to model (4) and consider the following gene network model in a vectormatrix form:

$$
\left\{\begin{array}{l}
\frac{\mathrm{d} m(t)}{\mathrm{d} t}=-A m(t)+W g(p(t-\tau))+l+G_{1} \omega(t) \\
\frac{\mathrm{d} p(t)}{\mathrm{d} t}=-B p(t)+D m(t-\sigma)+G_{2} \omega(t) \\
\tilde{z}(t)=\left[\begin{array}{c}
C_{1} m(t) \\
C_{2} p(t)
\end{array}\right]
\end{array}\right.
$$

where $m(t)=\left[m_{1}(t), m_{2}(t), \ldots, m_{n}(t)\right]^{T}, \quad p(t)=\left[p_{1}(t), p_{2}(t), \ldots\right.$, $\left.p_{n}(t)\right]^{T}$, the system parameters $A=\operatorname{diag}\left(a_{1}, a_{2}, \ldots, a_{n}\right)>0, B=$ $\operatorname{diag}\left(b_{1}, b_{2}, \ldots, b_{n}\right)>0$, and $D=\operatorname{diag}\left(d_{1}, d_{2}, \ldots, d_{n}\right)>0$ are diagonal matrices, $g(p(t))=\left[g_{1}\left(p_{1}(t)\right), g_{2}\left(p_{2}(t)\right), \ldots, g_{n}\left(p_{n}(t)\right)\right]^{T}$, and $l=\left[l_{1}\right.$, $\left.l_{2}, \ldots, l_{n}\right]^{T} . \omega(t)$ is the disturbance input which cannot be fully measured and are not completely known beforehand. We assume that $\omega(t)$ belongs to $L_{2}[0,+\infty)$, which implies that it is a function of finite energy. $G_{1}$ and $G_{2}$ are the input matrices, $C_{1}$ and $C_{2}$ are the output matrices, and $\tilde{z}(t)$ represents the concentration of mRNAs and proteins we are interested in.

Remark 1. It is worth pointing out that the theory developed in this paper can be generalized to the case when the time delays in (5) are different from each other. However, it is anticipated that the introduction of multiple time delays will result in more complicated notations, whereas no essential difficulty will be added.

Remark 2. The disturbance input $\omega(t)$ can be viewed as additive intrinsic and extrinsic signals that are not able to be detected. The output $\tilde{z}(t)$ can be thought as responses, if we are only interested in mRNAs or proteins, then we can let $\left[C_{1}, C_{2}\right]=[I, 0]$ or $\left[C_{1}, C_{2}\right]=[0, I]$, respectively. Particularly, if we are only interested in discussing the effect of disturbances on protein $i$, 
then we can just let $\left[C_{1}, C_{2}\right]=\left[0, e_{i}\right]$, where $e_{i}$ is the unit vector with every element of $e_{i}$ being 0 except 1 at the $i$ th element.

Let $\left(m^{*}, p^{*}\right)$ be the equilibrium point (steady state) of (5), that is, it is a solution of the following equations:

$$
\left\{\begin{array}{l}
0=-A m^{*}+W g\left(p^{*}\right)+l \\
0=-B p^{*}+D m^{*}
\end{array}\right.
$$

The aim of this paper is to investigate the influence of disturbance $\omega(t)$ on the stability of gene network (5). To achieve this, we will shift the steady state $\left(m^{*}, p^{*}\right)$ to the origin by using the transformation $x(t) \triangleq\left[x_{1}(t), x_{2}(t), \ldots, x_{n}(t)\right]^{T}=m(t)-m^{*}$ and $y(t) \triangleq\left[y_{1}(t)\right.$, $\left.y_{2}(t), \ldots, y_{n}(t)\right]^{T}=p(t)-p^{*}$, then we have

$$
\left\{\begin{array}{l}
\frac{\mathrm{d} x(t)}{\mathrm{d} t}=-A x(t)+W h(y(t-\tau))+G_{1} \omega(t) \\
\frac{\mathrm{d} y(t)}{\mathrm{d} t}=-B y(t)+D x(t-\sigma)+G_{2} \omega(t) \\
z(t)=\left[\begin{array}{l}
C_{1} x(t) \\
C_{2} y(t)
\end{array}\right]
\end{array}\right.
$$

where

$$
h(y(t))=\left[h_{1}\left(y_{1}(t)\right), h_{2}\left(y_{2}(t)\right), \ldots, h_{n}\left(y_{n}(t)\right)\right]^{T}
$$

with $h_{j}\left(y_{j}(t)\right)=g_{j}\left(y_{j}(t)+p_{j}^{*}\right)-g_{j}\left(p_{j}^{*}\right)$.

We assume the initial condition of the genetic regulatory network in (7) to be

$$
x(t)=\varphi_{x}(t), \quad y(t)=\psi_{y}(t), \quad-\varsigma \leq t \leq 0, \quad \varsigma=\max \{\tau, \sigma\} .
$$

where $\varphi_{x}$ and $\psi_{y}$ both belong to $\mathcal{C}\left([-\varsigma, 0], \mathbb{R}^{n}\right)$. Since $g_{i}$ is a monotonically increasing and differentiable function with saturation, it satisfies

$$
0 \leq \frac{g_{j}\left(s_{1}\right)-g_{j}\left(s_{2}\right)}{s_{1}-s_{2}} \leq k_{j}, \quad k_{j}>0, j \in \mathbb{Z}_{n}
$$

for any different $s_{1}, s_{2} \in \mathbb{R}$. From the relationship between $g$ and $h$, we obtain the following condition:

$$
h_{j}(s)\left(h_{j}(s)-k_{j} s\right) \leq 0, \quad j \in \mathbb{Z}_{n},
$$

for any $s \in \mathbb{R}$.

For convenience, we denote $K \triangleq \operatorname{diag}\left(k_{1}, k_{2}, \ldots, k_{n}\right)$ throughout the paper. In the following, we give the definition of disturbancelevel measurement from a nonlinear robust control point of view.

Definition 1. Network (7) is said to have attenuation level $\rho$, if it is globally asymptotically stable for $w(t)=0$, and under zero initial conditions $\varphi_{x}(t)=\psi_{y}(t)=0, t \in[-\varsigma, 0]$, it holds that

$$
\int_{0}^{T}|z(s)|^{2} \mathrm{~d} s \leq \rho^{2} \int_{0}^{T}|w(s)|^{2} \mathrm{~d} s,
$$

for any $T>0$ and nonzero input disturbance $w(t)$.

\section{Disturbance Attenuation of Genetic REGULATORY NETWORK}

The problem to be addressed in this section is to study the effect of external disturbances on the stability of gene networks (5) theoretically. Based on the analysis in Section 2, we study the stability of network (7) equivalently and present the main result in the following theorem:

Theorem 1. If there exist matrices $Q_{1}>0, Q_{2}>0, Q_{3}>0, Q_{4}>0$, $Q_{5}>0$, a diagonal matrix $\Gamma=\operatorname{diag}\left(\gamma_{1}, \gamma_{2}, \ldots, \gamma_{n}\right)>0$, and matrices $P_{11}, P_{12}, P_{22}, X_{1}, X_{2}, Y_{1}, Y_{2}$, such that the following LMIs hold:

$$
\begin{aligned}
\Upsilon(\bar{\tau}, \bar{\sigma}) & =\left[\begin{array}{cccc}
\Upsilon_{11} & \Upsilon_{12} & \Upsilon_{13} & \Upsilon_{14} \\
\# & \Upsilon_{22} & \Upsilon_{23} & \Upsilon_{24} \\
\# & \# & \Upsilon_{33} & \Upsilon_{34} \\
\# & \# & \# & \Upsilon_{44}
\end{array}\right]<0, \\
P & =\left[\begin{array}{cc}
P_{11} & P_{12} \\
\# & P_{22}
\end{array}\right]>0
\end{aligned}
$$

where

$$
\begin{aligned}
& \Upsilon_{11}=\left[\begin{array}{ccc}
M_{1} & P_{12} D+X_{1}+X_{2}^{T} & -A P_{12}-P_{12} B \\
\# & -Q_{1}-X_{2}-X_{2}^{T} & D P_{22} \\
\# & \# & M_{3}
\end{array}\right], \\
& \Upsilon_{12}=\left[\begin{array}{ccc}
0 & 0 & P_{11} W \\
D \Gamma & 0 & 0 \\
0 & Y_{1}+Y_{2}^{T} & P_{12}^{T} W
\end{array}\right], \\
& \Upsilon_{13}=\left[\begin{array}{ccc}
\bar{\sigma} X_{1} & 0 & P_{11} G_{1}+P_{12} G_{2} \\
-\bar{\sigma} X_{2} & 0 & 0 \\
0 & \bar{\tau} Y_{1} & P_{12}^{T} G_{1}+P_{22} G_{2}
\end{array}\right], \\
& \Upsilon_{14}=\left[\begin{array}{ccc}
\bar{\sigma} A Q_{4} & 0 \\
0 & -\bar{\tau} D Q_{5} \\
0 & \bar{\tau} B Q_{5}
\end{array}\right],
\end{aligned}
$$$$
\Upsilon_{22}=\operatorname{diag}\left(-2 K^{-1} \Gamma B+Q_{3},-Q_{2}-Y_{2}-Y_{2}^{T},-Q_{3}\right),
$$$$
\begin{aligned}
& \Upsilon_{23}=\left[\begin{array}{ccc}
0 & 0 & \Gamma G_{2} \\
0 & -\bar{\tau} Y_{2} & 0 \\
0 & 0 & 0
\end{array}\right], \\
& \Upsilon_{24}=\left[\begin{array}{ccc}
0 & 0 \\
0 & 0 \\
-\bar{\sigma} W^{T} Q_{4} & 0
\end{array}\right],
\end{aligned}
$$$$
\Upsilon_{33}=\operatorname{diag}\left(-\bar{\sigma} Q_{4}, \quad-\bar{\tau} Q_{5}, \quad-\rho^{2} I\right),
$$$$
\Upsilon_{34}=\left[\begin{array}{cc}
0 & 0 \\
0 & 0 \\
-\bar{\sigma} G_{1}^{T} Q_{4} & -\bar{\tau} G_{2}^{T} Q_{5}
\end{array}\right]
$$$$
\Upsilon_{44}=\operatorname{diag}\left(-\bar{\sigma} Q_{4}, \quad-\bar{\tau} Q_{5}\right),
$$

with

$$
\begin{aligned}
& M_{1}=-P_{11} A-A P_{11}-X_{1}-X_{1}^{T}+Q_{1}+C_{1}^{T} C_{1}, \\
& M_{3}=-P_{22} B-B P_{22}-Y_{1}-Y_{1}^{T}+Q_{2}+C_{2}^{T} C_{2},
\end{aligned}
$$

then the attenuation level $\rho$ of the perturbed genetic regulatory network in (7) is guaranteed for any $(\tau, \sigma)$ satisfying $\tau \leq \bar{\tau}$ and $\sigma \leq \bar{\sigma}$

From Theorem 1, one can see that we develop a sufficient condition under which the genetic regulatory network in (5) with $w(t)=0$ is globally asymptotically stable, and explore the mechanisms of gene networks to measure the disturbance level from the $L_{2}$ gain point of view. Also, for a preassigned disturbance level $\rho$, the condition in Theorem 1 is delay-dependent, which further implies that we can discuss the effect of time delays on the kinetics of gene networks and estimate the maximal delay pair $(\tau, \sigma)$ to ensure that the attenuation level $\rho$ of the perturbed genetic regulatory network in (7) is guaranteed by means of Theorem 1 . This will be of importance in revealing the impact of delays on the analysis of gene networks and introducing more flexibility in the design of delay-tolerant genetic circuit. 
Remark 3. Under conditions (11) and (12), one may prove the uniqueness of the equilibrium point by using a contradiction argument. To be specific, if there exists another equilibrium point $(\bar{m}, \bar{p})$ different from $\left(m^{*}, p^{*}\right)$, then by following the same analysis in Theorem 1 , one can prove that $(\bar{m}, \bar{p})$ is also globally asymptotically stable. Note that the conditions in Theorem 1 are independent of the equilibrium point; thus, there exist two globally asymptotically stable equilibria, which is impossible.

Remark 4. For fixed delay pair $(\bar{\tau}, \bar{\sigma})$, we may optimize the disturbance level $\rho$ by solving the following constrained optimization:

$$
\begin{aligned}
& \rho_{0} \triangleq \min _{S} \rho \\
& \text { subject to (11) and (12), }
\end{aligned}
$$

where $S=\left\{\rho>0, Q_{i}>0, i=1, \ldots, 5, \Gamma=\operatorname{diag}\left(\gamma_{1}, \gamma_{2}, \ldots, \gamma_{n}\right)>\right.$ $\left.0, P_{11}, P_{12}, P_{22}, X_{1}, X_{2}, Y_{1}, Y_{2}\right\}$. Note that the optimized value $\rho_{0}$ can be attained by a simple bisection algorithm [25].

Remark 5. In a real gene network, the output $z(t)$ may not be composed entirely of network state, but also be corrupted by the external disturbance $w(t)$. From the proof of Theorem 1, it can be seen that this will not add significant difficulty and similar results can also be obtained. In addition, one can show that if the initial condition $\varphi_{x}(t)$ and $\psi_{y}(t)$ are not zero, then an extra term should be added as follows:

$$
\int_{0}^{T}|z(s)|^{2} \mathrm{~d} s \leq \rho^{2} \int_{0}^{T}|w(s)|^{2} \mathrm{~d} s+V(0),
$$

for some positive-definite function $V(t)$.

Meanwhile, it is sometimes also the case that we may not know how long it will take for an individual process to be finished during the process of gene regulation. That is, we have no information about the magnitude of time delays in the signaling pathway, an interesting question one may raise is that whether we can still obtain a bit conservative condition, under which the attenuation level $\rho$ of the perturbed genetic regulatory network in (5) is guaranteed for any $\tau>0$ and $\sigma>0$. Similar to Theorem 1, we can obtain the so-called delay-independent condition on the disturbance analysis of gene networks (the proof is omitted here).

Theorem 2. If there exist matrices $Q_{1}>0, Q_{2}>0$, a diagonal matrix $\Gamma=\operatorname{diag}\left(\gamma_{1}, \gamma_{2}, \ldots, \gamma_{n}\right)>0$, and matrices $P_{11}, P_{12}, P_{22}$, such that the following LMIs hold:

$$
\begin{aligned}
& \Sigma=\left[\begin{array}{cc}
\Sigma_{11} & \Sigma_{12} \\
\# & \Sigma_{22}
\end{array}\right]<0, \\
& P=\left[\begin{array}{cc}
P_{11} & P_{12} \\
\# & P_{22}
\end{array}\right]>0,
\end{aligned}
$$

where

$$
\begin{aligned}
& \Sigma_{11}=\left[\begin{array}{ccc}
N_{11} & P_{12} D & -A P_{12}-P_{12} B \\
\# & -Q_{1} & D P_{22} \\
\# & \# & N_{33}
\end{array}\right] \\
& \Sigma_{12}=\left[\begin{array}{ccc}
0 & P_{11} W & P_{11} G_{1}+P_{12} G_{2} \\
D \Gamma & 0 & 0 \\
0 & P_{12}^{T} W & P_{12}^{T} G_{1}+P_{22} G_{2}
\end{array}\right] \text {, } \\
& \Sigma_{22}=\left[\begin{array}{ccc}
-2 K^{-1} \Gamma B+Q_{2} & 0 & \Gamma G_{2} \\
\# & -Q_{2} & 0 \\
\# & \# & -\rho^{2} I
\end{array}\right] \text {, }
\end{aligned}
$$

with

$$
\begin{aligned}
& N_{11}=-P_{11} A-A P_{11}+Q_{1}+C_{1}^{T} C_{1}, \\
& N_{33}=-P_{22} B-B P_{22}+C_{2}^{T} C_{2},
\end{aligned}
$$

then the attenuation level $\rho$ of the perturbed genetic regulatory network in (7) is guaranteed.

\section{Application to Three-Gene Networks}

In this section, we illustrate the application of the proposed results to a biological network, which has been adopted as a mathematical model, and experimentally studied in Escherichia coli [22]. The network is a cyclic negative feedback loop with three repressor genes (lacl, tet $R$, and $\mathrm{cl}$ ) and their corresponding promoters. Here, we incorporate time delays and external disturbances to the repressor system in [22] to check the stability of the steady state. Consider the following gene network described by six coupled differential equations:

$$
\left\{\begin{array}{l}
\frac{\mathrm{d} m_{i}(t)}{d t}=-m_{i}(t)+\alpha_{i} \frac{1}{1+p_{j}^{H}(t-\tau)}+\alpha_{0}+G_{1 i} \omega(t), \\
\frac{\mathrm{d} p_{i}(t)}{d t}=-\beta_{i} p_{i}(t)+\gamma_{i} m_{i}(t-\sigma)+G_{2 i} \omega(t),
\end{array}\right.
$$

where $i$ and $j$ have the following three pairs of values: $(i=1, j=2),(i=2, j=3),(i=3, j=1) . \beta_{i}$ denotes the ratio of the protein decay rate to the mRNA decay rate and $H$ is the Hill coefficient. From (15), we have

$$
\left\{\begin{aligned}
\frac{\mathrm{d} m_{i}(t)}{d t}= & -m_{i}(t)+\left(-\alpha_{i}\right) \frac{p_{j}^{H}(t-\tau)}{1+p_{j}^{H}(t-\tau)}+\alpha_{i} \\
& +\alpha_{0}+G_{1 i} \omega(t), \\
\frac{\mathrm{d} p_{i}(t)}{d t}= & -\beta_{i} p_{i}(t)+\gamma_{i} m_{i}(t-\sigma)+G_{2 i} \omega(t) .
\end{aligned}\right.
$$

Then, by selecting

$$
m(t)=\left[m_{1}(t), m_{2}(t), m_{3}(t)\right]^{T}, p(t)=\left[p_{1}(t), p_{2}(t), p_{3}(t)\right]^{T},
$$

and $g_{j}(x)=x^{2} /\left(1+x^{2}\right)$ for any $j$, we obtain network (5) with the following parameters:

$$
\begin{gathered}
A=\left[\begin{array}{lll}
1 & 0 & 0 \\
0 & 1 & 0 \\
0 & 0 & 1
\end{array}\right], \quad W=\left[\begin{array}{ccc}
0 & 0 & -\alpha_{1} \\
-\alpha_{2} & 0 & 0 \\
0 & -\alpha_{3} & 0
\end{array}\right], \\
B=\operatorname{diag}\left(\beta_{1}, \beta_{2}, \beta_{3}\right), \quad D=\operatorname{diag}\left(\gamma_{1}, \gamma_{2}, \gamma_{3}\right), \\
l=\left[\begin{array}{l}
\alpha_{1}+\alpha_{0} \\
\alpha_{2}+\alpha_{0} \\
\alpha_{3}+\alpha_{0}
\end{array}\right], \quad G_{1}=\left[\begin{array}{c}
G_{11} \\
G_{12} \\
G_{13}
\end{array}\right] \quad G_{2}=\left[\begin{array}{l}
G_{21} \\
G_{22} \\
G_{23}
\end{array}\right] .
\end{gathered}
$$

For numerical simulation, we choose the Hill coefficient $H=2$, then we have $K=\operatorname{diag}(0.65,0.65,0.65)$. Moreover, we assume that $\tau=1$ and $\sigma=2$, and the other parameters are taken as

$$
\begin{aligned}
\alpha_{1} & =0.8, \quad \alpha_{2}=0.6, \quad \alpha_{3}=0.6, \quad \alpha_{0}=0, \\
\beta_{1} & =\beta_{2}=\beta_{3}=1, \\
\gamma_{1} & =0.6, \quad \gamma_{2}=0.4, \quad \gamma_{3}=0.5, \\
G_{11} & =G_{13}=1, \quad G_{12}=0, \\
G_{21} & =G_{22}=1, \quad G_{23}=0 .
\end{aligned}
$$

By means of Theorem 1, it is easy to check that the genetic regulatory network in (15) is globally asymptotically stable when there are no external disturbances $(\omega(t)=0)$; thus, the steady state $m^{*}=(0.7389,0.5014,0.5766)^{T}, p^{*}=(0.4435,0.2008,0.2880)^{T}$ is a stable and unique equilibrium. Figs. 2 and 3 show the trajectory of the system state $m(t)$ and $p(t)$ with the initial state chosen as 


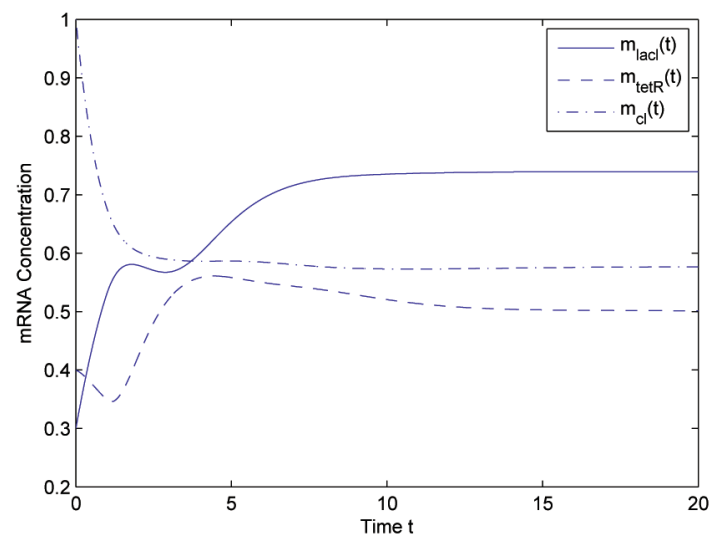

Fig. 2. State response of $m(t)$ of genetic regulatory network in (15) with $w(t)=0$.

$\varphi(t)=[0.3+0.5 \sin t, 0.2+0.2 \cos t, 1.0-0.6 \sin t]^{T} \quad$ a n d $\quad \psi(t)=$ $[0.5+0.5 \cos t, 0.4-0.2 \sin t, 0.8-0.4 \cos t]^{T}$.

Furthermore, if we are interested in the effects of additive disturbance $\omega(t)$ on individual protein $p_{1}, p_{2}$, and $p_{3}$, then we can let $C$ in (5) be $[1,0,0],[0,1,0]$, and $[0,0,1]$, respectively. By Remark 4, we solve the LMIs in (11) and (12) to calculate the attenuation level and obtain the optimal attenuation level $\rho_{0}$ of protein $p_{1}, p_{2}$, and $p_{3}$, as $0.7432,0.4684$, and 0.4630 via Yalmip [26], respectively, which further implies that the disturbance attenuation levels of these proteins cannot exceed these corresponding scalars. Equivalently, we have

$$
\begin{aligned}
& \int_{0}^{T}\left|p_{1}(s)-p_{1}^{*}\right|^{2} \mathrm{~d} s \leq 0.5523 \int_{0}^{T}|w(s)|^{2} \mathrm{~d} s, \\
& \int_{0}^{T}\left|p_{2}(s)-p_{2}^{*}\right|^{2} \mathrm{~d} s \leq 0.2194 \int_{0}^{T}|w(s)|^{2} \mathrm{~d} s, \\
& \int_{0}^{T}\left|p_{3}(s)-p_{3}^{*}\right|^{2} \mathrm{~d} s \leq 0.2143 \int_{0}^{T}|w(s)|^{2} \mathrm{~d} s .
\end{aligned}
$$

Therefore, in this gene network, it can be concluded that the disturbances at $p_{1}, p_{2}$, and $p_{3}$ are all attenuated by the network, which was verified by the proposed theory. For numerical simulation, we assume that $w(t)=2 /(1+0.5 t)$, and the initial condition as $\varphi(t)=[0.5,0.2,0.4]^{T}$ and $\psi(t)=[0.1,0.2,0.3]^{T}$. Fig. 4 depicts the dynamic response of the perturbed genetic regulatory network in (15), where the solid lines show the state trajectory of protein under the excitation of external disturbances, and the dashed lines illustrate the steady state of the undisturbed gene network.

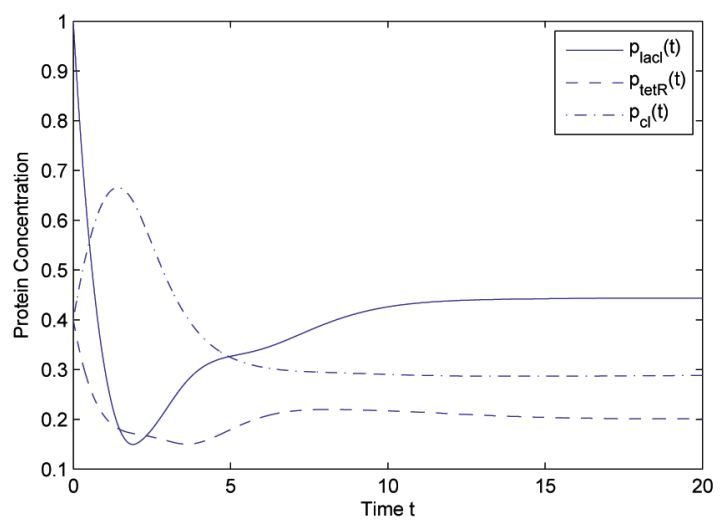

Fig. 3. State response of $p(t)$ of genetic regulatory network in (15) with $w(t)=0$.
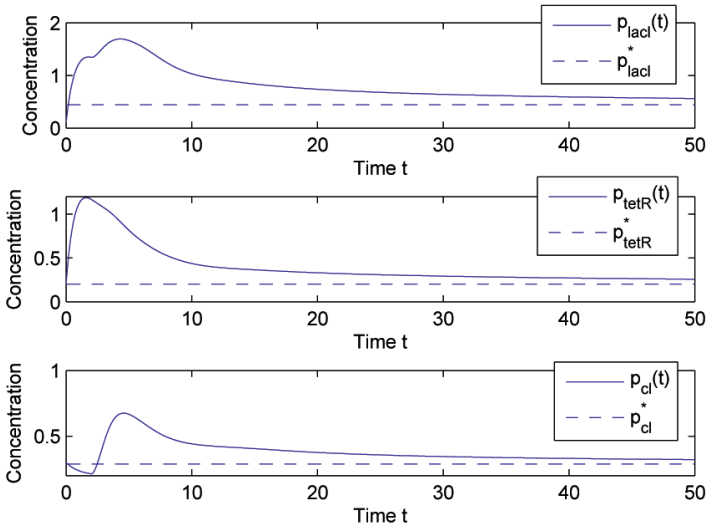

Fig. 4. State response of $p(t)$ of perturbed genetic regulatory network in (15).

\section{Conclusion}

In this study, a nonlinear differential equation model of delayed genetic regulatory network was proposed, and the dynamic behaviors of such a class of networks were investigated. To be precise, we presented the stability conditions for gene networks with both time delays and external disturbances, and explored the mechanisms of gene networks to measure the disturbance level from the signal processing perspective. Moreover, we presented a convex optimization algorithm to minimize the disturbance level by virtue of $L_{2}$ control theory. To verify the obtained theoretical results, a three-gene network example was used for numerical illustration.

\section{APPENDIX}

\section{Proof of Theorem 1}

According to Definition 1, we show that the attenuation level $\rho$ of the perturbed genetic regulatory network in (7) is guaranteed in two steps. First, we prove that the genetic regulatory network in (7) with $w(t)=0$ is globally asymptotically stable under the condition (11), and then, we show that (10) is satisfied under zero initial conditions.

Step I. It follows from (11) that

$$
\Omega(\bar{\tau}, \bar{\sigma})=\left[\begin{array}{cc}
\Omega_{11} & \Omega_{12} \\
\# & \Omega_{22}
\end{array}\right]<0,
$$

where

$$
\begin{aligned}
& \Omega_{11}=\left[\begin{array}{cc}
\Omega_{111} & \Omega_{112} \\
\# & \Omega_{113}
\end{array}\right], \\
& \Omega_{111}=\left[\begin{array}{ccc}
\tilde{M}_{1} & P_{12} D+X_{1}+X_{2}^{T} & -A P_{12}-P_{12} B \\
\# & -Q_{1}-X_{2}-X_{2}^{T} & D P_{22} \\
\# & \# & \tilde{M}_{3}
\end{array}\right] \text {, } \\
& \Omega_{112}=\left[\begin{array}{ccc}
0 & 0 & P_{11} W \\
D \Gamma & 0 & 0 \\
0 & Y_{1}+Y_{2}^{T} & P_{12}^{T} W
\end{array}\right] \text {, } \\
& \Omega_{113}=\operatorname{diag}\left(-2 K^{-1} \Gamma B+Q_{3},-Q_{2}-Y_{2}-Y_{2}^{T},-Q_{3}\right) \text {, } \\
& \Omega_{12}=\left[\begin{array}{cccc}
\bar{\sigma} X_{1} & 0 & \bar{\sigma} A Q_{4} & 0 \\
-\bar{\sigma} X_{2} & 0 & 0 & -\bar{\tau} D Q_{5} \\
0 & \bar{\tau} Y_{1} & 0 & \bar{\tau} B Q_{5} \\
0 & 0 & 0 & 0 \\
0 & -\bar{\tau} Y_{2} & 0 & 0 \\
0 & 0 & -\bar{\sigma} W^{T} Q_{4} & 0
\end{array}\right] \text {, } \\
& \Omega_{22}=\operatorname{diag}\left(-\bar{\sigma} Q_{4},-\bar{\tau} Q_{5},-\bar{\sigma} Q_{4},-\bar{\tau} Q_{5}\right) \text {, }
\end{aligned}
$$


with

$$
\begin{aligned}
& \tilde{M}_{1}=-P_{11} A-A P_{11}-X_{1}-X_{1}^{T}+Q_{1}, \\
& \tilde{M}_{3}=-P_{22} B-B P_{22}-Y_{1}-Y_{1}^{T}+Q_{2} .
\end{aligned}
$$

For convenience, we denote $x(t-\sigma)$ and $y(t-\tau)$ as $x_{\sigma}(t)$ and $y_{\tau}(t)$, respectively. Choose the Lyapunov functional candidate of the form

$$
V(t)=\sum_{i=1}^{4} V_{i}(t, x(t), y(t)),
$$

where

$$
\begin{aligned}
V_{1}(t, x(t), y(t))= & {\left[\begin{array}{l}
x(t) \\
y(t)
\end{array}\right]^{T} P\left[\begin{array}{l}
x(t) \\
y(t)
\end{array}\right], } \\
V_{2}(t, x(t), y(t))= & 2 \sum_{i=1}^{n} \gamma_{i} \int_{0}^{y_{i}(t)} h_{i}(\alpha) \mathrm{d} \alpha, \\
V_{3}(t, x(t), y(t))= & \int_{t-\sigma}^{t} x^{T}(\alpha) Q_{1} x(\alpha) \mathrm{d} \alpha \\
& +\int_{t-\tau}^{t} y^{T}(\alpha) Q_{2} y(\alpha) \mathrm{d} \alpha \\
& +\int_{t-\tau}^{t} h^{T}(y(\alpha)) Q_{3} h(y(\alpha)) \mathrm{d} \alpha, \\
V_{4}(t, x(t), y(t))= & \int_{-\sigma}^{0} \int_{t+\beta}^{t} \dot{x}^{T}(\alpha) Q_{4} \dot{x}(\alpha) \mathrm{d} \alpha \mathrm{d} \beta \\
& +\int_{-\tau}^{0} \int_{t+\gamma}^{t} \dot{y}^{T}(\alpha) Q_{5} \dot{y}(\alpha) \mathrm{d} \alpha \mathrm{d} \gamma .
\end{aligned}
$$

For techniques on the choice of Lyapunov functional, we refer the readers to [27] for details. To facilitate the stability analysis of gene network (7), we first calculate the time derivative of $V_{i}(t, x(t), y(t))$ along the trajectory of network (7), we have

$$
\begin{aligned}
\dot{V}_{1}(t, x, y)= & 2\left(x^{T}(t) P_{11}+y^{T} P_{12}^{T}\right) \dot{x}(t) \\
& +2\left(x^{T}(t) P_{12}+y^{T}(t) P_{22}\right) \dot{y}(t), \\
\dot{V}_{2}(t, x, y)= & 2 h^{T}(y(t)) \Gamma \dot{y}(t) \\
= & -2 h^{T}(y(t)) \Gamma B y(t)+2 h^{T}(y(t)) \Gamma D x_{\sigma}(t), \\
\dot{V}_{3}(t, x, y)= & x^{T}(t) Q_{1} x(t)-x_{\sigma}^{T}(t) Q_{1} x_{\sigma}(t) \\
& +y^{T}(t) Q_{2} y(t)-y_{\tau}^{T}(t) Q_{2} y_{\tau}(t) \\
& +h^{T}(y(t)) Q_{3} h(y(t))-h^{T}\left(y_{\tau}(t)\right) Q_{3} h\left(y_{\tau}(t)\right), \\
\dot{V}_{4}(t, x, y)= & \int_{-\sigma}^{0}\left[\dot{x}^{T}(t) Q_{4} \dot{x}(t)-\dot{x}^{T}(t+\beta) Q_{4} \dot{x}(t+\beta)\right] \mathrm{d} \beta \\
& +\int_{-\tau}^{0}\left[\dot{y}^{T}(t) Q_{5} \dot{y}(t)-\dot{y}^{T}(t+\gamma) Q_{5} \dot{y}(t+\gamma)\right] \mathrm{d} \gamma \\
= & \int_{t-\sigma}^{t}\left[x^{T}(t) A Q_{4} A x(t)-2 x^{T}(t) A Q_{4} W h\left(y_{\tau}(t)\right)\right. \\
& \left.+h^{T}\left(y_{\tau}(t)\right) W^{T} Q_{4} W h\left(y_{\tau}(t)\right)-\dot{x}^{T}(\beta) Q_{4} \dot{x}(\beta)\right] \mathrm{d} \beta \\
& +\int_{t-\tau}^{t}\left[y^{T}(t) B Q_{5} B y(t)-2 y^{T}(t) B Q_{5} D x_{\sigma}(t)\right. \\
& \left.+x_{\sigma}^{T}(t) D Q_{5} D x_{\sigma}(t)-\dot{y}^{T}(\gamma) Q_{5} \dot{y}(\gamma)\right] \mathrm{d} \gamma .
\end{aligned}
$$

Considering the relationship in (9) and noting $\Gamma>0$, we can deduce

$$
-2 h^{T}(y(t)) \Gamma B y(t) \leq-2 h^{T}(y(t)) \Gamma B K^{-1} h(y(t)) .
$$

In addition, for any matrices $X_{1}$ and $X_{2}$, we have

$$
\begin{aligned}
& 2 x^{T}(t) X_{1} \int_{t-\sigma}^{t} \dot{x}(\beta) \mathrm{d} \beta \\
& \quad=2 x^{T}(t) X_{1} x(t)-2 x^{T}(t) X_{1} x_{\sigma}(t), \\
& 2 x_{\sigma}^{T}(t) X_{2} \int_{t-\sigma}^{t} \dot{x}(\beta) \mathrm{d} \beta \\
& \quad=2 x_{\sigma}^{T}(t) X_{2} x(t)-2 x_{\sigma}^{T}(t) X_{2} x_{\sigma}(t) .
\end{aligned}
$$

Likewise, for any matrices $Y_{1}$ and $Y_{2}$, we have

$$
\begin{aligned}
& 2 y^{T}(t) Y_{1} \int_{t-\tau}^{t} \dot{y}(\gamma) \mathrm{d} \gamma \\
& \quad=2 y^{T}(t) Y_{1} y(t)-2 y^{T}(t) Y_{1} y(t-\tau), \\
& 2 y_{\tau}^{T}(t) Y_{2} \int_{t-\tau}^{t} \dot{y}(\gamma) \mathrm{d} \gamma \\
& \quad=2 y_{\tau}^{T}(t) Y_{2} y(t)-2 y_{\tau}^{T}(t) Y_{2} y_{\tau}(t) .
\end{aligned}
$$

It then follows from (22)-(30) that

$$
\begin{aligned}
\dot{V}(t) & =\sum_{i=1}^{4} \dot{V}_{i}(t, x(t), y(t)) \\
& \leq \frac{1}{\tau \sigma} \int_{t-\tau}^{t} \int_{t-\sigma}^{t} \eta^{T}(t, \beta, \gamma) \Xi(\tau, \sigma) \eta(t, \beta, \gamma) \mathrm{d} \beta \mathrm{d} \gamma,
\end{aligned}
$$

where

$$
\begin{aligned}
\eta(t, \beta, \gamma)= & {\left[x^{T}(t), x_{\sigma}^{T}(t), y^{T}(t), h^{T}(y(t)), y_{\tau}^{T}(t),\right.} \\
& \left.h^{T}\left(y_{\tau}(t)\right), \dot{x}^{T}(\beta), \dot{y}^{T}(\gamma)\right]^{T} \\
\Xi(\tau, \sigma)= & {\left[\begin{array}{ccc}
\Omega_{11} & \sigma \varrho_{1}^{T} & \tau \varrho_{2}^{T} \\
\# & -\sigma Q_{4} & 0 \\
\# & \# & -\tau Q_{5}
\end{array}\right] } \\
+ & \sigma \chi_{1}^{T} Q_{4}^{-1} \chi_{1}+\tau \chi_{2}^{T} Q_{5}^{-1} \chi_{2}
\end{aligned}
$$

and

$$
\begin{aligned}
\varrho_{1} & =\left[\begin{array}{llllll}
X_{1}^{T} & -X_{2}^{T} & 0 & 0 & 0 & 0
\end{array}\right], \\
\varrho_{2} & =\left[\begin{array}{llllllll}
0 & 0 & Y_{1}^{T} & 0 & -Y_{2}^{T} & 0
\end{array}\right], \\
\chi_{1} & =\left[\begin{array}{llllllll}
Q_{4} A & 0 & 0 & 0 & 0 & -Q_{4} W & 0 & 0
\end{array}\right], \\
\chi_{2} & =\left[\begin{array}{llllllll}
0 & -Q_{5} D & Q_{5} B & 0 & 0 & 0 & 0 & 0
\end{array}\right] .
\end{aligned}
$$

Define

$$
\begin{aligned}
\kappa_{1} & =\left[\begin{array}{cccccc}
X_{1}^{T} & -X_{2}^{T} & 0 & 0 & 0 & 0 \\
Q_{4} A & 0 & 0 & 0 & 0 & -Q_{4} W
\end{array}\right], \\
\kappa_{2} & =\left[\begin{array}{cccccc}
0 & 0 & Y_{1}^{T} & 0 & -Y_{2}^{T} & 0 \\
0 & -Q_{5} D & Q_{5} B & 0 & 0 & 0
\end{array}\right],
\end{aligned}
$$

then

$$
\Omega_{11}+\sigma \kappa_{1}^{T} \operatorname{diag}\left(Q_{4}^{-1}, Q_{4}^{-1}\right) \kappa_{1}+\tau \kappa_{2}^{T} \operatorname{diag}\left(Q_{5}^{-1}, Q_{5}^{-1}\right) \kappa_{2}
$$

is a monotonic increasing matrix function with respect to $\sigma$ and $\tau$, respectively. Moreover, based on Schur Complement equivalence in [28] and (16), we have $\Xi(\tau, \sigma)<0$, which further indicates that $\dot{V}(t, x(t), y(t))<0$ for any nonzero $\eta(t, \beta, \gamma)$ in (31). Thus, it follows from [29] that the genetic regulatory network in (7) with $w(t)=0$ is globally asymptotically stable.

Step II. We show that $z(t)$ in (7) satisfies $\int_{0}^{\infty}|z(t)|^{2} \mathrm{~d} t \leq$ $\rho^{2} \int_{0}^{\infty}|w(t)|^{2} \mathrm{~d} t$. For $\rho>0$ and $T>0$, we define

$$
J(T) \triangleq \int_{0}^{T}\left[|z(t)|^{2}-\rho^{2}|w(t)|^{2}\right] \mathrm{d} t .
$$


Observing the fact that

$$
0=\int_{0}^{T} \frac{\mathrm{d} V(t)}{\mathrm{d} t} \mathrm{~d} t+V(0)-V(T),
$$

where $\frac{\mathrm{d} V(s)}{\mathrm{d} s}$ is the time derivative along the trajectory of network (7), we have

$$
\begin{aligned}
& J(T)=\int_{0}^{T}\left[|z(t)|^{2}-\rho^{2}|w(t)|^{2}\right] \mathrm{d} t \\
& +\int_{0}^{T} \frac{\mathrm{d} V(t)}{\mathrm{d} t} \mathrm{~d} t+V(0)-V(T) \\
& =\int_{0}^{T}\left[|z(t)|^{2}-\rho^{2}|w(t)|^{2}\right] \mathrm{d} t \\
& +\int_{0}^{T}\left[2\left(x^{T}(t) P_{11}+y^{T}(t) P_{12}^{T}\right)\right. \\
& \left.\times\left(-A x(t)+W h\left(y_{\tau}(t)\right)+G_{1} \omega(t)\right)\right] \mathrm{d} t \\
& +\int_{0}^{T}\left[2\left(x^{T}(t) P_{12}+y^{T}(t) P_{22}\right)\right. \\
& \left.\times\left(-B y(t)+D x_{\sigma}(t)+G_{2} \omega(t)\right)\right] \mathrm{d} t \\
& +\int_{0}^{T}\left[-2 h^{T}(y(t)) \Gamma B y(t)+2 h^{T}(y(t)) \Gamma D x_{\sigma}(t)\right. \\
& \left.+2 h^{T}(y(t)) \Gamma G_{2} \omega(t)\right] \mathrm{d} t \\
& +\int_{0}^{T}\left[x^{T}(t) Q_{1} x(t)-x_{\sigma}^{T}(t) Q_{1} x_{\sigma}(t)\right] \mathrm{d} t \\
& +\int_{0}^{T}\left[y^{T}(t) Q_{2} y(t)-y_{\tau}^{T}(t) Q_{2} y_{\tau}(t)\right] \mathrm{d} t \\
& +\int_{0}^{T}\left[h^{T}(y(t)) Q_{3} h(y(t))-h^{T}\left(y_{\tau}(t)\right) Q_{3} h\left(y_{\tau}(t)\right)\right] \mathrm{d} t \\
& +\int_{0}^{T} \int_{t-\sigma}^{t}\left[\dot{x}^{T}(t) Q_{4} \dot{x}(t)-\dot{x}^{T}(\beta) Q_{4} \dot{x}(\beta)\right] \mathrm{d} \beta \mathrm{d} t \\
& +\int_{0}^{T} \int_{t-\tau}^{t}\left[\dot{y}^{T}(t) Q_{5} \dot{y}(t)-\dot{y}^{T}(\gamma) Q_{5} \dot{y}(\gamma)\right] \mathrm{d} \gamma \mathrm{d} t \\
& +V(0)-V(T) \text {. }
\end{aligned}
$$

Since the initial values $\varphi_{x}$ and $\psi_{y}$ are assumed to be zero, then $V(0)=0$. In addition, $V(T) \geq 0, \forall T>0$. Using a similar method in Step I, we obtain from (11) that $J(T)<0, \forall T>0$. That is, the attenuation level $\rho$ of perturbed genetic regulatory network in (7) is guaranteed, and the proof is thus completed.

\section{ACKNOWLEDGMENTS}

The authors would like to thank the editor-in-chief, the associate editor, and the anonymous reviewers for their constructive comments and suggestions to improve the quality of the paper. The work was partially supported by GRF HKU 7138/10E.

\section{RefERENCES}

[1] N. Wiener, Cybernetics of Control and Communication in the Animal and the Machine. The MIT Press, 1948.

[2] P. Smolen, D.A. Baxter, and J.H. Byrne, "Mathematical Modeling of Gene Networks," Neuron, vol. 26, no. 3, pp. 567-580, 2000

[3] H. de Jong, "Modeling and Simulation of Genetic Regulatory Systems: A Literature Review," J. Computational Biology, vol. 9, pp. 67-103, 2002.

[4] C.J. Tomlin and J.D. Axelrod, "Biology by Numbers: Mathematical Modelling in Developmental Biology," Nature Rev. Genetics, vol. 8, pp. 331-340, 2007.

[5] T. Schlitt and A. Brazma, "Current Approaches to Gene Regulatory Network Modelling," BMC Bioinformatics, vol. 8, no. suppl 6, p. s9, 2007.

[6] P. D’haeseleer, S. Liang, and R. Somogyi, "Genetic Network Inference: From Co-Expression Clustering to Reverse Engineering," Bioinformatics, vol. 16, no. 8, pp. 707-726, 2000.
[7] O. Wolkenhauer and M. Mesarović, "Feedback Dynamics and Cell Function: Why Systems Biology Is Called Systems Biology," Molecular BioSystems, vol. 1, no. 1, pp. 14-16, 2005.

[8] L. Chen and K. Aihara, "Stability of Genetic Regulatory Networks with Time Delay," IEEE Trans. Circuits and Systems (I), vol. 49, no. 5, pp. 602-608, May 2002.

[9] D. Angeli, J.E. Ferrell, and E.D. Sontag, "Detection of Multistability Bifurcations, and Hysteresis in a Large Class of Biological PositiveFeedback Systems," Proc. Nat'l Academy of Sciences USA, vol. 101, no. 7, pp. 1822-1827, 2004.

[10] A. Becskei and L. Serrano, "Engineering Stability in Gene Networks by Autoregulation," Nature, vol. 405, no. 6786, pp. 590-593, 2000.

[11] H. Hirata, S. Yoshiura, T. Ohtsuka, Y. Bessho, T. Harada, K. Yoshikawa, and R. Kageyama, "Oscillatory Expression of the bHLH Factor Hes1 Regulated by a Negative Feedback Loop," Science, vol. 298, no. 5594, pp. $840-843,2002$.

[12] E. Klipp, R. Herwig, A. Kowald, C. Wierling, and H. Lehrach, Systems Biology in Practice: Concepts, Implementation and Application. Wiley-VCH, 2005.

[13] N.A.M. Monk, "Oscillatory Expression of Hes1, p53, and NF- $\kappa$ B Driven by Transcriptional Time Delays," Current Biology, vol. 13, no. 16, pp. 1409-1413, 2003.

[14] C. Li, L. Chen, and K. Aihara, "Stability of Genetic Networks with SUM Regulatory Logic: Lur'e System and LMI Approach," IEEE Trans. Circuits and Systems (I), vol. 53, no. 11, pp. 2451-2458, Nov. 2006.

[15] C.H. Yuh, H. Bolouri, and E.H. Davidson, "Genomic Cis-Regulatory Logic: Experimental and Computational Analysis of a Sea Urchin Gene," Science, vol. 279, no. 5358, pp. 1896-1902, 1998.

[16] Y. Setty, A.E. Mayo, M.G. Surette, and U. Alon, "Detailed Map of a CisRegulatory Input Function," Proc. Nat'l Academy of Sciences USA, vol. 100, no. 13 , pp. 7702-7707, 2003.

[17] E.H. Snoussi, "Qualitative Dynamics of Piecewise-Linear Differential Equations: A Discrete Mapping Approach," Dynamical and Stability of Systems, vol. 4, no. 3, pp. 565-583, 1989.

[18] C. Li, L. Chen, and K. Aihara, "Stochastic Stability of Genetic Networks with Disturbance Attenuation," IEEE Trans. Circuits and Systems (II), vol. 54, no. 10 , pp. 892-896, Oct. 2007.

[19] B. Chen and Y. Wang, "On the Attenuation and Amplification of Molecular Noise in Genetic Regulator Networks," BMC Bioinformatics, vol. 7, no. 1, p. 52, 2006.

[20] E.D. Sontag, “Molecular Systems Biology and Control," European J. Control, vol. 11, no. 5, pp. 396-435, 2005.

[21] V.S. Vaidhyanathan, Regulation and Control Mechanisms in Biological Systems. PTR Prentice Hall, 1993.

[22] M.B. Elowitz and S. Leibler, "A Synthetic Oscillatory Network of Transcriptional Regulators," Nature, vol. 403, no. 6767, pp. 335-338, 2000.

[23] S. Kalir and U. Alon, "Using a Quantitative Blueprint to Reprogram the Dynamics of the Flagella Gene Network," Cell, vol. 117, no. 6, pp. 713-720, 2004

[24] N.E. Buchler, U. Gerland, and T. Hwa, "On Schemes of Combinatorial Transcription Logic," Proc. Nat'l Academy of Sciences USA, vol. 100, no. 9, pp. 5136-5141, 2003.

[25] S. Boyd and L. Vandenberghe, Convex Optimization. Cambridge Univ. Press, 2004.

[26] J. Löfberg, "Yalmip: A Toolbox for Modeling and Optimization in MATLAB," Proc. IEEE Int'l Symp. Computer-Aided Control System Design (CACSD), 2004.

[27] S. Xu and J. Lam, "A Survey of Linear Matrix Inequality Techniques in Stability Analysis of Delay Systems," Int'l J. Systems Science, vol. 39, no. 12, pp. 1095-1113, 2008.

[28] S. Boyd, L.E. Ghaoui, E. Feron, and U. Balakrishnana, Linear Matrix Inequalities in System and Control Theory. SIAM, 1994.

[29] J.K. Hale and S.M.V. Lunel, Introduction to Functional Differential Equations. Springer, 1993.

$\triangleright$ For more information on this or any other computing topic, please visit our Digital Library at www.computer.org/publications/dlib. 